\title{
Bioabsorbable devices in spinal and cranial neurosurgery
}

\author{
Charles L. Branch JR., M.D. \\ Department of Neurosurgery, Wake Forest University School of Medicine, Winston-Salem, North Carolina
}

Bioabsorbable devices in surgery have a long history of use in wound closure and to some degree maxillofacial reconstruction. The potential utility of bioabsorbable devices in structural support applications in the spine has been the focus of recent research in Europe and the US. Preclinical experience coupled with improved polymer chemistry and lengthy experience in maxillofacial applications has driven recent clinical investigation in spinal applications as well.

In this issue of Neurosurgical Focus, Robbins and colleagues present a review of accumulated published research with bioabsorbable devices in spinal applications. This report also summarizes the purported benefits of bioabsorbable devices over conventional metallic implants.

Optimization of radiographic assessment of fusion is one of the proposed benefits of bioabsorbable devices. Krijnen and colleagues from Amsterdam report their experience with magnetic resonance (MR) imaging in a preclinical model. This experience appears to support the potential role of MR imaging in assessment of fusion and of the surrounding tissue after treatment with a bioabsorbable device.

More recent experience in the preclinical and clinical settings is reported by investigators from the US and Europe. Not only are structural support applications being considered, but potential applications as a bone graft or biological container and as a protective adhesion barrier are being investigated. Early experience with these applications is reported as well.

Lippman and colleagues, including the late David Cahill, report their preclinical experience in a cervical interbody fusion model. This documents the results of the use of two different bioabsorbable compounds combined with conventional autograft bone or recombinant human bone morphogenetic protein-2 (rhBMP-2) in a caprine cervical spine model. Early clinical experience with a bioabsorbable interbody anterior cervical fusion device is reported by Vaccaro, et al., who used demineralized bone matrix as the graft material, and by Lanman and Hopkins, who used rhBMP-2. These reports affirm the potential role of these compounds in this application but represent a very shortterm evaluation in two small series of patients.

The use of a bioabsorbable anterior cervical fixation or graft containment plate and screw construct is investigated in the ovine model by Cornwall and colleagues. This construct was associated with a satisfactory fusion rate without device-related complications in this animal model.
Clinical experience with lumbar interbody fusion is reported in three manuscripts. These represent both early and more long-term experience in small series of patients. Early experience with a unilateral interbody fusion technique (transforaminal lumbar interbody fusion) in which a bioabsorbable spacer and rhBMP-2 were used as the fusion induction material, is reported by Lanman and Hopkins in 43 patients with a 10-month mean follow-up duration, and by Kuklo, et al., in 22 patients with a 12month mean follow up. Although these reports document very early follow up according to conventional fusion analysis expectations, in both series computerized tomography is used to document what appears to be a satisfactory fusion at this early follow-up interval without devicerelated complications. Couture and Branch report their experience with a bilateral interbody fusion technique (posterior lumbar interbody fusion) in which locally harvested bone autograft was used in patients with a mean 18-month follow-up duration. Although this report documents fusion rates comparable to those in previously reported series in which metallic or nonabsorbable synthetic devices were used, cavitation defects associated with pseudarthrosis warrant further investigation. It appears that insufficient interbody graft material or endplate preparation are more likely to result in symptomatic pseudarthrosis as the bioabsorbable device resorbs.

A novel application of a bioabsorbable film as an adhesion barrier in spinal surgery is reported by Klopp, Welch, and colleagues. This preclinical experience in the ovine laminectomy model appears to hold promise for the prevention of dural adhesions after decompressive spinal surgery.

Experience with these compounds in craniofacial applications continues to evolve. Cohen and colleagues reported on the safety and efficacy of a more rapidly reabsorbing compound in a pediatric population. Liu, et al., report their experience with a bioactive product in the repair of cranial defects. Although the material in their study is not a bioabsorbable compound, their experience represents the bridge between the inert nonabsorbable devices and those that have apparent biological activity even if they are not truly bioabsorbable.

The role of bioabsorbable devices will continue to evolve and will be clarified with further investigation. This compilation of articles represents another step toward an understanding of the role that these devices will play in the treatment of both spinal and cranial disease. 